\title{
Rinsing Processes in Open-width Washing Machines
}

\section{A B J Kroezen, $H \mathrm{~J} L \mathrm{~J}$ van der Linden and J Groot Wassink}

Chemical Engineering Department

Twente University of Technology

PO Box 217

7500 AE Enschede

The Netherlands

A simulator is described for rinsing processes carried out on open-width washing machines. In combination with a theoretical model, a simple method is given for testing rinsing processes. The method has been used to investigate the extraction of caustic soda from a cotton fabric, varying the temperature, fabric velocity and type of fabric. The results were verified by measurements in a commercial open-width washing machine. The absorption of carbon dioxide from the atmosphere in the washing water is shown to be important for removing the caustic soda. The method may be used in practical situations to optimise process conditions.

\section{INTRODUCTION}

After a fabric has undergone several different treatments during textile finishing, impurities must be removed from it, extraction being a very commonly used method. There are different standard methods of processing depending on the form of the fabric, e.g. open width, rope or beam. The washing process we shall discuss here is one which is carried out in the open-width washing machine. In earlier work [1] a general process was described for rinsing processes in open-width washing machines. Although an overall process description can be used, all rinsing processes differ in detail on points such as temperature, fabric velocity, rinsing ratio and length and geometry of the open-width washing machine. Moreover operation and optimisation of the different variables are different for each rinsing process, so that a suitable method for defining the rinsing process is desirable.

We present here details of a simulator comprising one compartment of an open-width washing machine that enables the rinsing process to be quantified. In combination with the so-called E-NTU model [1] it is possible to predict the efficiency of the washing process.

The simulator for the open-width washing machine has been used for a study of the extraction of caustic soda after

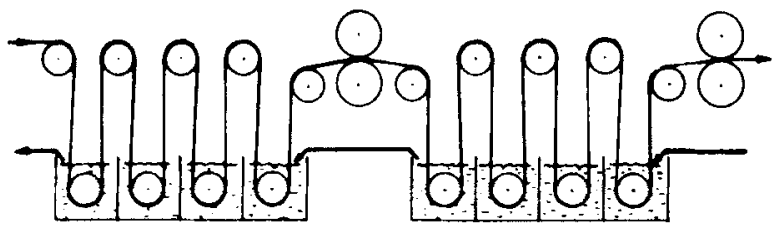

Open-width washing machine mercerisation. The results were verified by experiments on a commercial scale.

\section{PROCESS DESCRIPTION}

The E-NTU model is a commonly used process description for several types of heat and mass exchangers [2]. The open-width washing machine will be considered as a counter-current extractor with plug-flow conditions (see Figures 1 and 2).

The following mass-transfer equations hold for fabric flow (Eqn 1) and wash water flow (Eqn 2):

$$
\begin{aligned}
m \phi_{\mathrm{d}} \frac{\partial c}{\partial x} & =k \frac{\partial A}{\partial x}((c / m)-\gamma) \\
\phi_{\mathrm{b}} \frac{\partial \gamma}{\partial x} & =k \frac{\partial A}{\partial x}((c / m)-\gamma)
\end{aligned}
$$

Boundary conditions are defined in Eqns 3 and 4 for fabric and wash water respectively:

$$
\begin{array}{ll}
x=0 & c=c_{0} \\
x=L & \gamma=\gamma_{0}=0
\end{array}
$$

The overall mass-transfer coefficient $(K)$ can be written in terms of the relevant partial mass-transfer coefficients (Eqn 5):

$$
\frac{1}{K}=\frac{1}{K_{1} m}+\frac{1}{K_{2}}
$$

where $m$ is the distribution coefficient between the fibre and the water phase, $K_{1}$ is the partial mass-transfer coefficient in the fibre and $K_{2}$ is the partial mass-transfer coefficient in the water phase.

The solution of the Eqns 1 and 2 with respect to the boundary conditions (Eqns 3 and 4) may be written as Eqn 6:

$$
\frac{c}{c_{\mathrm{o}}}=1-\frac{1-\exp \left(-N_{\mathrm{TU}}(1-1 / F)\right)}{1-(1 / F)\left(\exp \left(-N_{\mathrm{TU}}(1-1 / F)\right)\right)}
$$

where $\quad N_{\mathrm{TU}}=K A / m \phi_{\mathrm{d}}$

$$
F=\phi_{\mathrm{b}} / m \phi_{\mathrm{d}}
$$

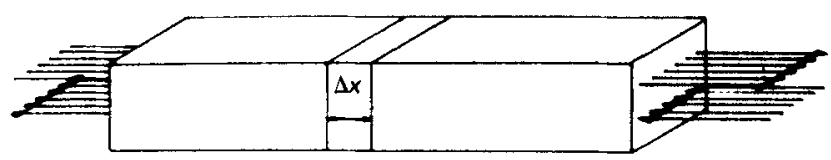

Countercurrent extractor with plug-flow conditions 


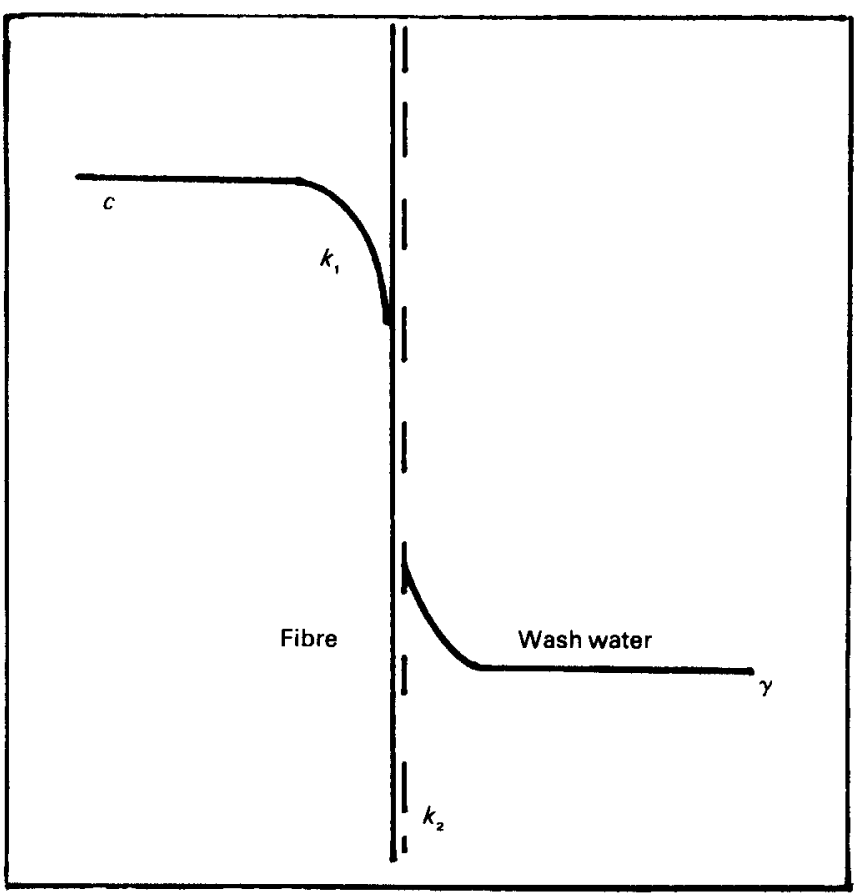

Figure 2-Mass transfer in an open-width washing machine

Eqn 6 gives the efficiency $E\left(=1-\left(c / c_{0}\right)\right)$ for a countercurrent extractor with plug-flow conditions expressed in terms of two parameters: $F$ and $N_{\text {TU }}$.

In open-width washing machines with vertical fabric transport the amount of wash water normally does not affect the mass-transfer coefficient. The influence of wash-water consumption on process efficiency can be determined using Eqn 6.

\section{PROCESS SIMULATION}

The basic unit of an open-width washing machine is a compartment. All physical phenomena taking place in the open-width washing machine are present in such a unit. Therefore the simulator chosen was effectively a onecompartment open-width washing machine (see Figures 3 and 4).

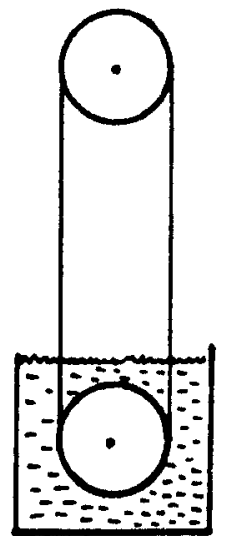

Simulator (n revolutions

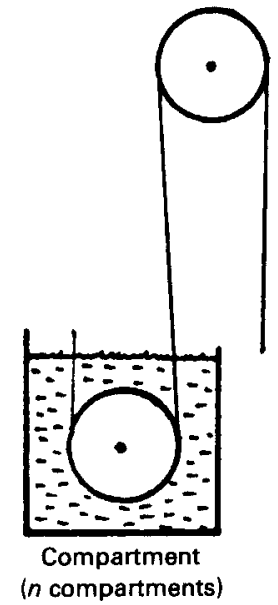

Figure 3 - Analogy between simulator and compartment

The concentration of the component to be extracted can be measured by:

(a) Continuous measurement of the conductivity in the wash water (salts, dyes)

(b) Continuous measurement by spectrometry in the fabric $[3 \mid$ or in the wash water (dyes)

(c) Sampling the fabric after $n$ revolutions and analysing the samples afterwards [4].

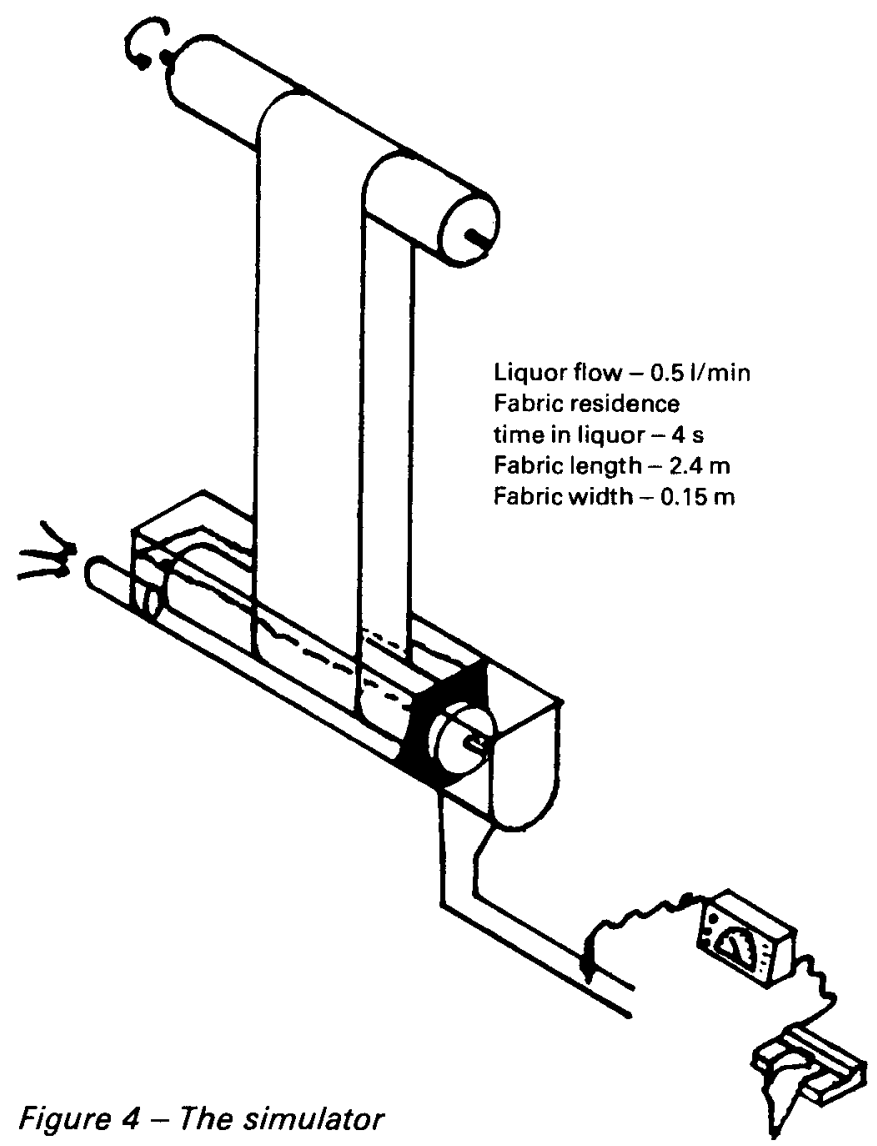

The first alternative was chosen for the rinsing experiments.

A rinsing curve may be obtained by:

(a) Fixing the impregnated fabric on the simulator

(b) Revolving the fabric under fixed process conditions (fabric velocity, temperature, fabric tension, etc.)

(c) Starting the measurement by filling the bath instantaneously and arranging a constant wash water flow.

By integrating the various time intervals of the differential concentration signal a rinsing curve can be calculated (Figure 5).

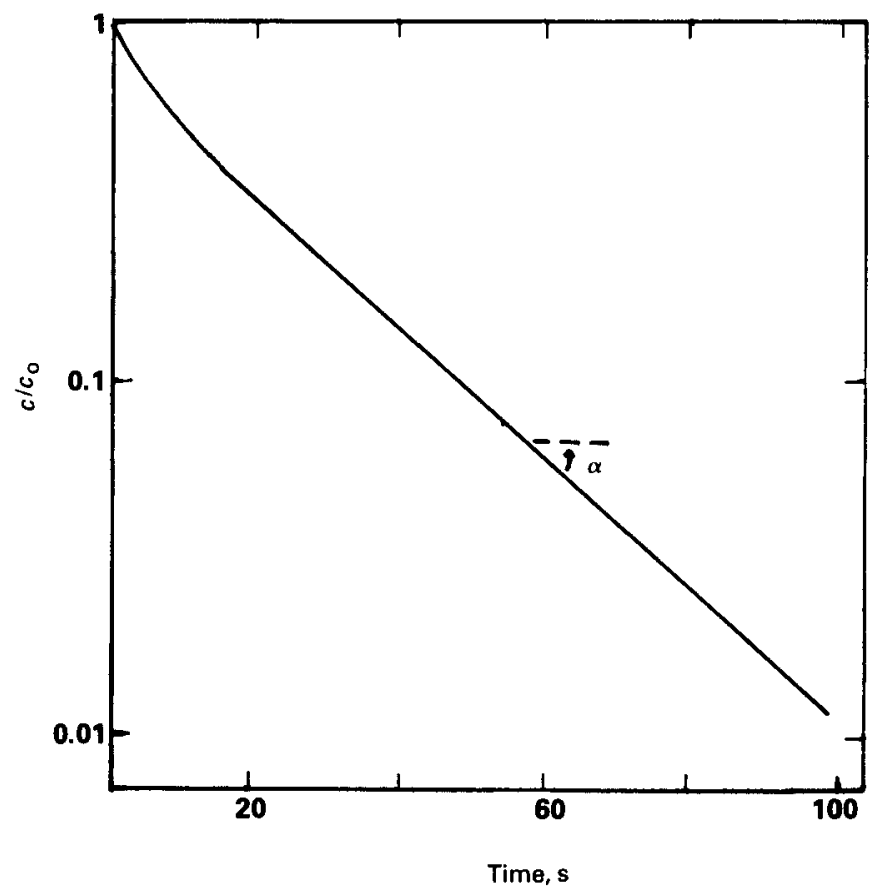

Figure 5-Example of a rinsing curve 
The rinsing process is simulated using a very large rinsing ratio $(F \rightarrow \infty)$. This simplifies Eqn 6 of the E-NTU model to Eqn 7:

$$
\frac{c}{c_{0}}=1-\exp \left(-N_{\mathrm{TU}}\right)=1-\exp \left(-n_{\mathrm{TU}}^{*} \tau\right)
$$

Actual open-width washing machines with a finite rinsing ratio can be described by Eqn 6 using this experimental $N_{\text {TU }}$ value.

\section{SIMULATION EXPERIMENTS: EXTRACTION OF CAUSTIC SODA}

Simulation experiments were carried out on the extraction of caustic soda after mercerisation. The extraction has been studied as a function of several process parameters such as fabric velocity, temperature, type of fabric and number of revolutions. All fabrics were impregnated in a $220 \mathrm{~g} / \mathrm{l}$ solution of caustic soda.

\section{Mass Transfer as a Function of Temperature and Fabric Velocity}

The rinsing process was studied at fabric velocities from 10 to $100 \mathrm{~m} / \mathrm{min}$ and temperatures in the range 20 to $80^{\circ} \mathrm{C}$. The rinsing curve becomes linear after a certain period. The non-linear part at the start of the curve is due to nonstationary mass transfer as well as to deswelling effects of the fabric (see Figures 6 and 7 ).

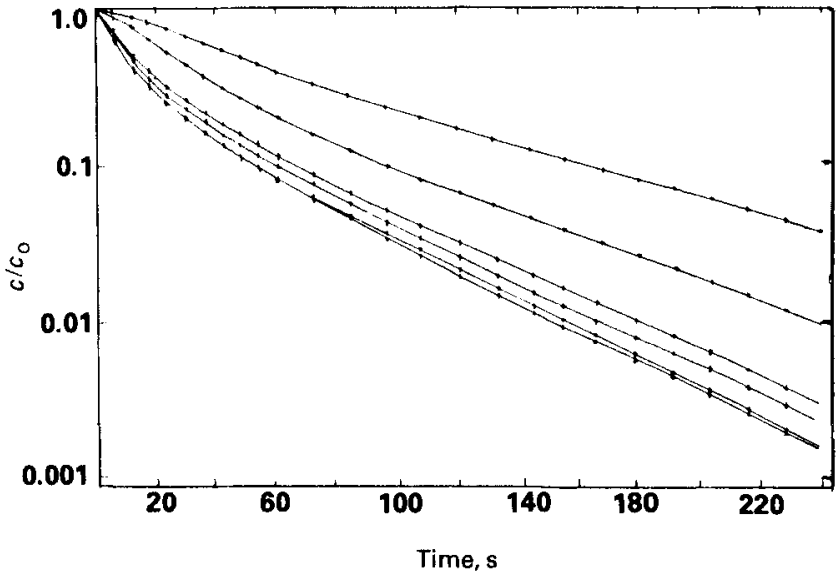

Figure 6 - Rinsing curves at varying fabric velocities 10 (top curve), 20, 40, 80 and 100 (bottom curve) $\mathrm{m} / \mathrm{min}$

\section{Rinsing Velocity for Different Types of Fabric}

Three different types of fabric have been tested at a temperature of $20^{\circ} \mathrm{C}$. Mass transfer depends on the type of fabric, as can be seen in Figure 8, the mass-transfer rate for open thin fabrics being much higher than for thicker fabrics.

It is quite difficult to correlate mass transfer and type of fabric directly. Besides fabric thickness, fabric structure and yarn porosity have a definite effect on mass transfer. For example, from Figure 8 it seems that under the same process conditions poplin needs twice as long in an openwidth machine to be washed with the same efficiency as the Java print fabric.

\section{MECHANISM OF MASS TRANSFER}

Because of the strong affinity between caustic soda and cotton, the fabric absorbs a large quantity of caustic soda during mercerisation. In the rinsing process the caustic soda must be transported from the intrafibre voids to the bath liquor. In this study we have tried to analyse and quantify this mechanism of transport.

The compartment of the open-width washing machine

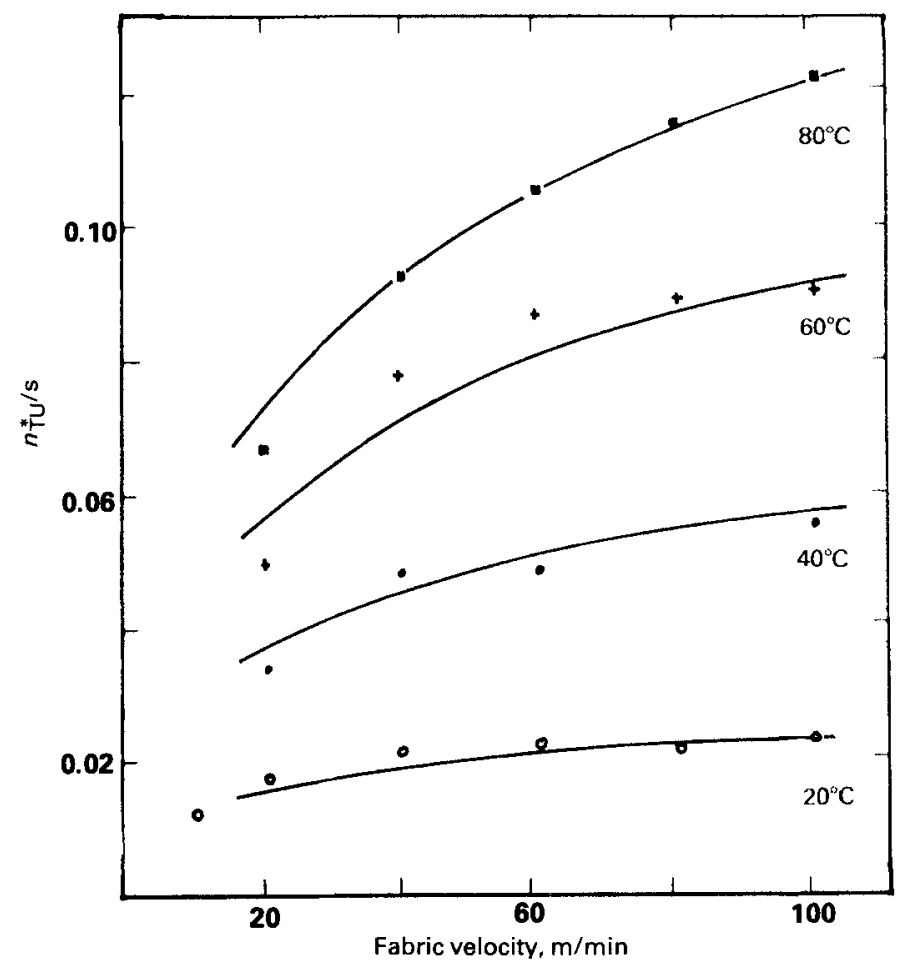

Figure 7 - Values of $\mathrm{n}_{T U}^{*}$ at varying temperatures and fabric velocities

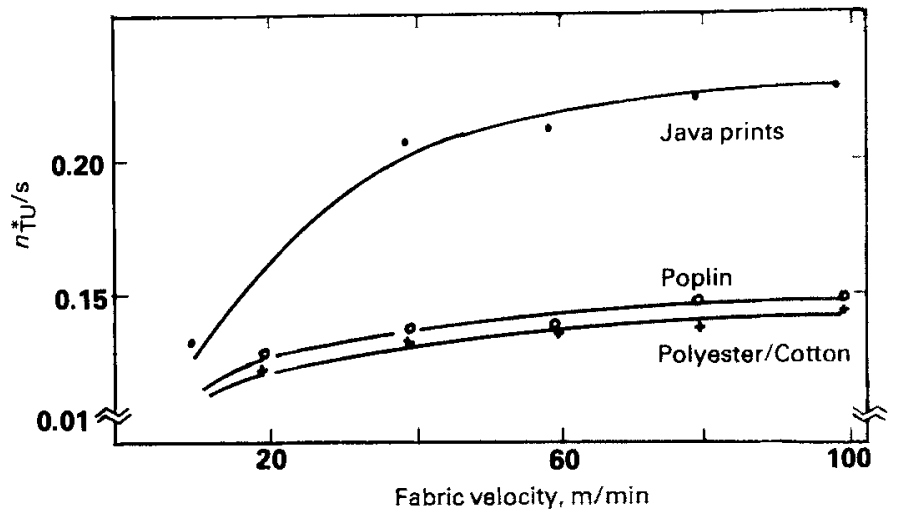

Figure 8 -Values of $\mathrm{n}_{\text {Tu }}^{*}$ for different types of fabric

can be separated into two sections: section 1 is the part above the bath liquor and section 2 is the part in the wash water (see Figure 9). In section 1 an exchange of caustic soda between the entrained film and the fabric takes place. Caustic soda is transported by diffusion from the fibres to the entrained film. In section 2 the entrained film will be removed by fresh wash water supplied by convective transport. The overall resistance to mass transfer, which can be represented by an overall mass-transfer coefficient, is the sum of a series of partial mass-transfer resistances (Figure 10).

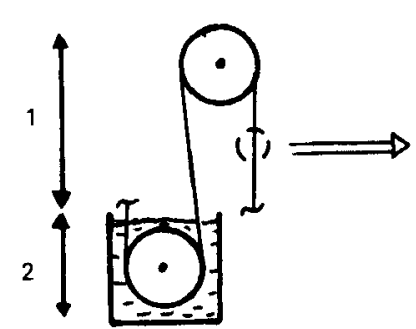

1 - Section 1

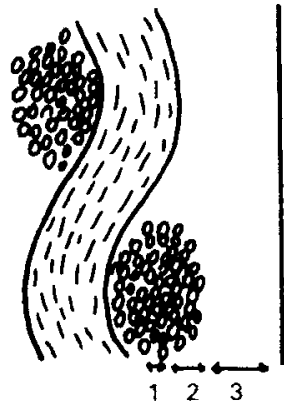

2 - Section 2

Figure 9 -Analysis of two different mass-transfer sections in a compartment 

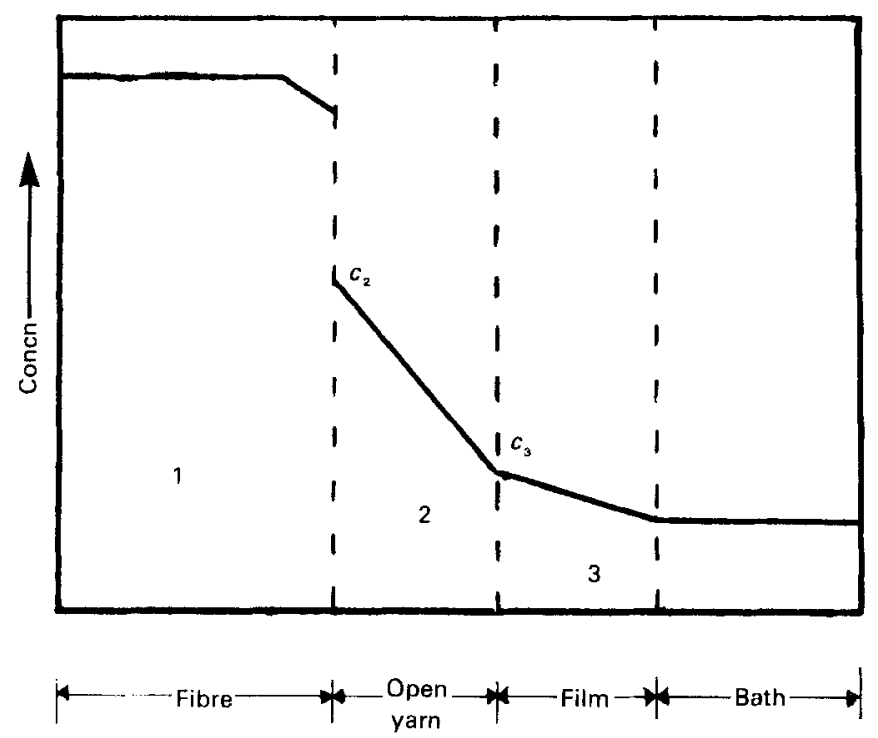

$K_{v}=$ mass-transfer coefficient in the fibre (1)

$m=$ distribution coefficient $\left(=c_{1} / c_{2}\right)$

$K_{\mathrm{d}}=$ mass transfer coefficient in the open yarn voids (2)

$K_{f}=$ mass-transfer coefficient in the film (3)

Figure 10 -Model of mass transfer

Assuming a complete series arrangement of the masstransfer resistances, the relationship shown in Eqn 8 for the mass transfer is valid:

$$
\begin{aligned}
\phi & =K A\left(c-\frac{\gamma}{m}\right)=K_{\mathrm{v}} A_{\mathrm{v}}\left(c-c_{1}\right)=K_{\mathrm{d}} A_{\mathrm{d}}\left(\frac{c_{2}}{m}-\frac{c_{3}}{m}\right) \\
& =K_{\mathrm{f}}\left(\frac{c_{3}}{m}-\frac{\gamma}{m}\right)
\end{aligned}
$$

where $m=c_{1} / c_{2}$

so that Eqn 9 can be written:

$$
K=\left(\frac{m A}{K_{\mathrm{v}} A_{v}}+\frac{1}{K_{\mathrm{d}}}+\frac{1}{K_{\mathrm{f}}}\right)^{-1}
$$

giving rise to the expression in Eqn 10 for $n_{\mathrm{TU}}^{*}$

$$
n_{\mathrm{T} U}^{*}=\frac{k A}{\phi_{\mathrm{d}} m} \cdot \frac{1}{\tau}=\frac{K}{m \delta}=\frac{1}{m \delta}\left(\frac{m A}{K_{\mathrm{v}} A_{\mathrm{v}}}+\frac{1}{K_{\mathrm{d}}}+\frac{1}{K_{\mathrm{f}}}\right)^{-1}
$$

The overall mass-transfer resistance is the sum of three partial resistances arranged in series. Assuming the resistance in the fibre is negligible, as found in practice, Eqn 9 simplifies to Eqn 11:

$$
K=\left(\frac{1}{K_{\mathrm{d}}}+\frac{1}{K_{\mathrm{f}}}\right)^{-1} \text { and } n_{\mathrm{TU}}^{*}=\frac{1}{m \delta}\left(\frac{1}{K_{\mathrm{d}}}+\frac{1}{K_{\mathrm{f}}}\right)^{-1}
$$

\section{Open-yarn Resistance}

Mass transfer in the open yarn is mainly determined by diffusion. The corresponding coefficient $\left(K_{d}\right)$ may be represented by Eqn 12:

$$
K_{\mathrm{d}} \sim D / \delta
$$

\section{Resistance of Entrained Film}

The resistance in the entrained film is determined firstly by diffusion in the film during the exchange period and secondly by removal of the film in a compartment by bath liquor. This process can be described in terms of the socalled penetration theory. The mass-transfer coefficient $\left(K_{f}\right)$ is proportional to the square root of the diffusion coefficient $D$ divided by the time of revolution $t$ (Eqn 13):

$$
K_{\mathrm{f}} \sim(D / t)^{\frac{1}{2}}
$$

The time of revolution is the ratio of fabric length per compartment to fabric velocity (Eqn 14):

$$
t \sim L / V
$$

\section{Distribution Coefficient}

In extraction processes cotton exhibits a typical sorption behaviour. The distribution coefficient $(m)$ differs for adsorption and desorption processes, and is dependent on the concentration. Vieweg [5], and Chedin and Marsaudon [6] have quoted adsorption distribution coefficients of $0.7-0.8(\mathrm{~mol} / \mathrm{g} / \mathrm{mol} / \mathrm{g})$ at $20^{\circ} \mathrm{C}$.

In our work a hysteresis in the adsorption-desorption distribution coefficients was noticed. The desorption distribution coefficient falls from about $2(\mathrm{~mol} / \mathrm{g} / \mathrm{mol} / \mathrm{g})$ at $20^{\circ} \mathrm{C}$ to 1 at $85^{\circ} \mathrm{C} \mathrm{[7].} \mathrm{The} \mathrm{relationship} \mathrm{in} \mathrm{Eqn} 15$ gives a satisfactory result in describing the extraction of caustic soda from a fabric:

$$
n_{\mathrm{TU}}^{*}=\frac{1}{m \delta}\left(\frac{\delta}{7.0 D}+0.28\left(\frac{L}{D v}\right)^{\frac{1}{2}}\right)^{-1}
$$

The curves given in Figure 7 represent the situation described by Eqn 15.

In Figure 11 the desorption distribution coefficients at $40^{\circ} \mathrm{C}$ and $60^{\circ} \mathrm{C}$ respectively have been calculated according to Eqn 15 by interpolation between the values at 20 and $85^{\circ} \mathrm{C}$.

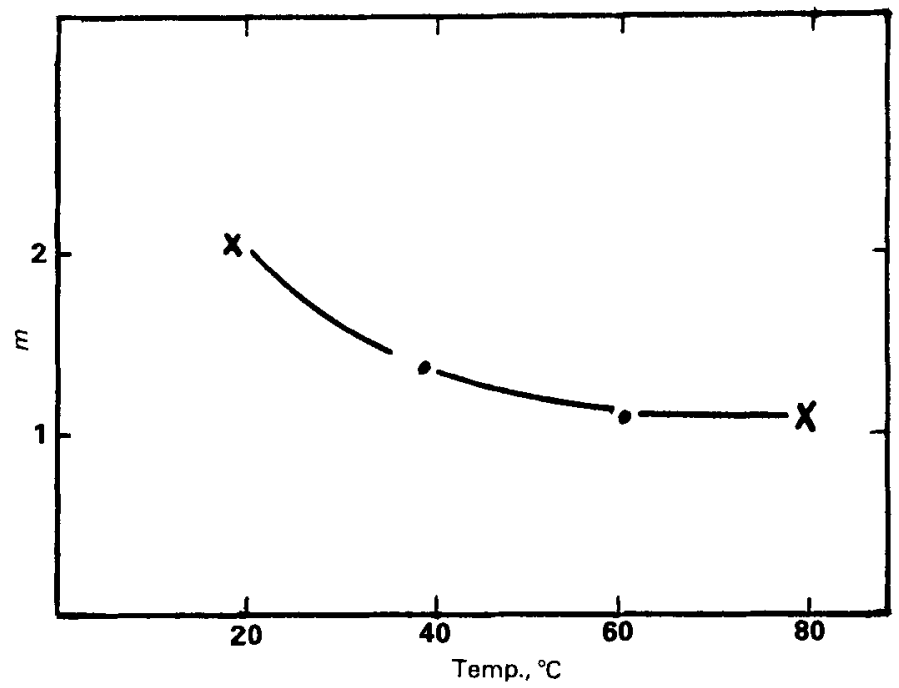

Figure 11 -Desorption distribution coefficient as a function of temperature

\section{COMMERCIAL-SCALE EXPERIMENTS}

Experiments on a commercial open-width washing machine have been carried out under stationary process conditions to verify the results of the simulation experiments. The open-width washing machine consisted of three washing vessels, each containing five compartments. 
Fabric and liquor samples were taken from every compartment, and were analysed for hydroxide, bicarbonate and carbonate ions. The total quantity of base in the fabric and bath is given in Figure 12.

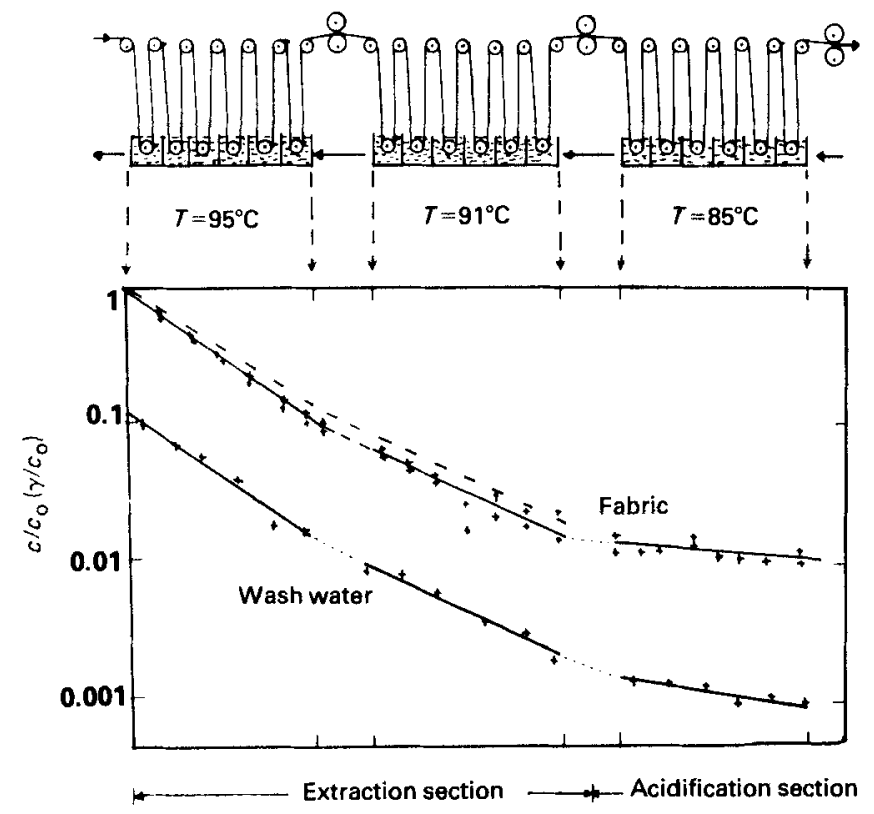

Figure 12 - Total base concentration in the open-width washing machine (dashed line is simulation result)

From the experiments it can be concluded that the open-width washing process can be separated into two sections, as follows.

1. Extraction section, taking place in the first two vessels, where the caustic soda is extracted as expected. The results are in good agreement with the simulator results.

2. Acidification section, taking place in the last vessel, where the caustic soda is no longer extracted but converted to carbonate by carbon dioxide absorbed by and present in the wash water (Scheme 1). The $\mathrm{pH}$ of the fabric decreases very fast in this part of the washing machine.

$$
\begin{gathered}
\mathrm{CO}_{2}+2 \mathrm{OH}^{-} \rightarrow \mathrm{OH}^{-}+\mathrm{HCO}_{3}^{-} \rightarrow \mathrm{CO}_{3}^{2-}+\mathrm{H}_{2} \mathrm{O} \\
\text { Scheme } 1
\end{gathered}
$$

In the first section $99 \%$ of the caustic soda is extracted $(\mathrm{pH}$ decreases from 14 to 12 ) and in the second section the remaining caustic soda is converted to carbonate $(\mathrm{pH}$ decreases from 12 to 9) (see Figure 13). In common practice the purified fabric is only tested at a required $\mathrm{pH}$ of approximately 9 . Therefore the acidification section is an essential part of the rinsing process to reach a low $\mathrm{pH}$ value.

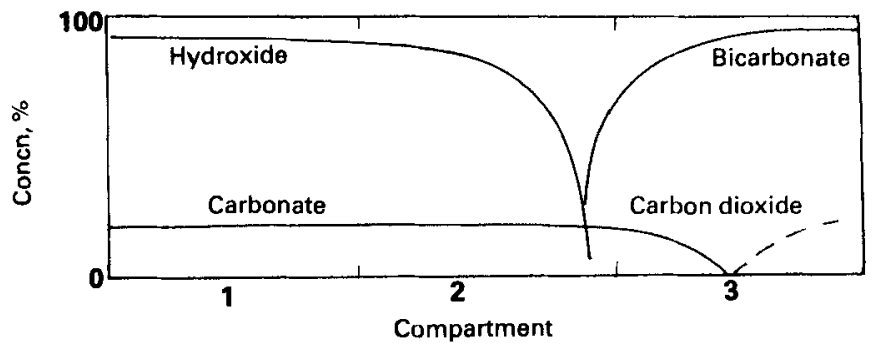

Figure 13 - Composition of the base in a series of three washing vessels

\section{CONCLUSIONS}

A simulator of an open-width washing machine has been found to give a simple method for testing rinsing processes. Using the E-NTU model the results of the simulator operating at an infinite rinsing ratio can be extrapolated to an actual rinsing ratio as might be found in an open-width washing machine. In future the simulator could be of great help in optimising the process variables and the design of open-width washing machines.

The extraction of caustic soda on an industrial scale has been studied as a function of several process variables. A verification of simulator experiments gave fairly good results. Substantial deviations take place if, apart from extraction, other phenomena are important, e.g. acidification reactions.

\section{REFERENCES}

1. J Groot Wassink, H J L J van der Linden, Text. Research J., 53 (1983) 751.

2. $W M$ Kays and $A L$ London, 'Compact Heat Exchangers' (New York: McGraw Hill, 1964).

3. R Faken, J Groot Wassink and H J L J van der Linden, Textiltechnik, 33 (1983) 41.

4. R B Holweg, Proc. 11th IFATCC Congress, Venice (June 1978).

5. W Vieweg, Ber., 40 (1907) 3876.

6. J Chedin and A Marsaudon, Makromolekulaire Chemie, 15 (1955) 115

7. H J L J van der Linden, J Groot Wassink and H J Vos, Text. Research J., 54 (1984) 77.

\section{LIST OF SYMBOLS}

\section{Symbol Description}

A Mass-transfer surface $\mathrm{m}^{2}$

c, $\gamma \quad$ Fabric and bath concentrations $\mathrm{mol} \mathrm{g}^{-1}$

$D \quad$ Effective diffusion coefficient $\mathrm{m}^{2} \mathrm{~s}^{-1}$

$E \quad$ Rinsing efficiency or porosity

$F \quad$ Rinsing ratio

$k \quad$ Mass-transfer coefficient

$L \quad$ Fabric length

$m \quad$ Distribution coefficient

$\mathrm{m} \mathrm{s}_{\mathrm{s}^{-1}}$

$N_{\text {TU }} \quad$ Overall number of transfer units

$n$ TU $\quad N_{\text {TU }} / \tau$

$\tau$ Residence time s

$T$ Temperature $\mathrm{K}$

$v \quad$ Fabric velocity $\mathrm{m} \mathrm{s}^{-1}$ or $\mathrm{m} \mathrm{min}^{-1}$

$w \quad$ Specific fabric weight $\mathrm{g} \mathrm{m}^{-2}$

$\phi_{\mathrm{d}}, \phi_{\mathrm{b}} \quad$ Fabric and bath-liquor flow rates $\mathrm{m}^{3} \mathrm{~s}^{-1}$

$\partial$ Fabric thickness $\mathrm{m}$

$\phi$ Mass flow $\mathrm{mol} \mathrm{s}^{-1}$ 